\title{
Pom-meling heart disease in mice
}

A glass of pomegranate juice (PG) a day may keep the cardiologist away, according to a new study in mice, in which Italian and American scientists demonstrated that mice drinking the juice had significantly reduced atherosclerosis.

Atherosclerosis, or hardening of the arteries, results when oxidative stress causes damage to the artery endothelium, leading to a buildup of fatty deposits, cholesterol, calcium and other substances on the artery wall. As these lesions, or plaques, grow they impede blood flow; a ruptured plaque can cause a blood clot that can result in a heart attack or stroke, depending on the site of the rupture. A diet high in antioxidant-rich fruits and vegetables may retard the development and progression of atherosclerosis.

The pomegranate (Punica granatum) is a peculiar-looking fruit, characterized by hundreds of seeds, each surrounded by juicy flesh. The fruit, which the ancient Chinese associated with longevity, has had a surge in popularity due in part to the availability of its vitamin- and antioxidant-rich juice in grocery stores. Until now, the artery-protecting effects of PG had remained untested.

Now, in the 29 March issue of the Proceedings of the National Academy of Sciences USA, a group led by Claudio Napoli of the University of Naples (Italy) provides evidence that regular consumption of PG can indeed have a role in preventing and treating atherosclerotic lesions. The group tested the preventive and therapeutic effects of PG in male hypercholesterolemic low-density-lipoprotein receptor-deficient $\left(\mathrm{LDLR}^{-/}\right)$mice fed a high-cholesterol diet. They allowed one group of mice to develop plaques and then put them on the PG regimen; they fed the other group PG beginning at a young age. In both groups, atherosclerotic lesions were $\sim 20 \%$ smaller in the PG-treated mice as compared with controls.

Napoli's group also tested the effects of PG supplementation on cultured human coronary artery endothelial cells that were exposed to high levels of shear stress and found that the PG-treated cells produced significantly more artery-protecting nitric oxide (NO) than controls. As research team member Lou Ignarro of UCLA explains, "[PG]

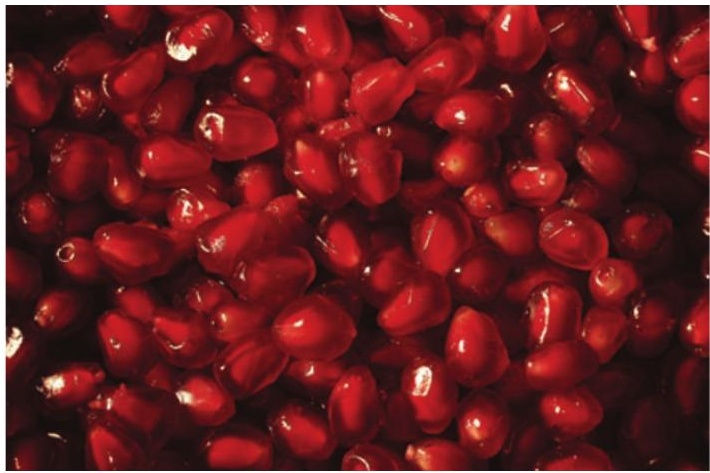

works by protecting NO against destruction [by free radicals], thereby increasing the functional levels of NO in the arteries. This leads to lower blood pressure and prevention of atherosclerosis."

The next step in this work, Ignarro tells Lab Animal, is to "conduct a clinical trial in humans to determine if daily consumption of pomegranate juice can reduce the severity of atherosclerosis by improving blood flow and reducing the plaque buildup in the affected arteries."

Tanja Schub

\section{RATS HAVE A SNACK, HOLD THE COKE}

The brain may have different circuits for handling the perceived rewards from drugs as opposed to healthier passions, such as food, suggesting new potential strategies for the treatment of addiction.

In the novel $A$ Clockwork Orange, a medical treatment designed to prevent an ultra-violent teenager from committing acts of aggression unexpectedly renders him incapable of listening to classical music. Though a fictional example, this highlights a serious concern inherent in developing therapies for psychiatric disorders-keeping a treatment focused and minimizing unexpected side effects. A new study from Nature Neuroscience (April) offers potentially important new findings in this area, revealing that it may be possible to therapeutically diminish the 'reward' associated with addictive drugs without affecting reward signals resulting from other stimuli.

Previous studies have shown that a brain structure called the subthalamic nucleus (STN) is involved in processing reward pathway signaling in response to certain stimuli-including drugs of addiction. In an effort to examine more closely the role of this brain region, Christelle Baunez of the Université de Provence (Marseilles, France) and her colleagues chemically induced STN lesions in rats, then observed the impact of these lesions on the animals' behavior in a variety of studies designed to measure the effort that rats would devote to obtaining food pellets or cocaine.
In experiments in which a single press of a bar yielded a food or cocaine reward, both the experimental and control rats behaved similarly. However, when the study was changed so that each reward event required an increased amount of effort (i.e., more than one press of the bar), it became clear that control rats were willing to put in significantly more effort to obtain cocaine rewards. Conversely, in those same studies, the experimental rats proved more willing to work hard toward a food reward.

Follow-up studies measuring 'place-conditioning' - the amount of time that an animal spends in an environment associated with a specific reward-mirrored these findings, showing that rats with STN lesions showed a reduced tendency to linger in a cocaine-associated compartment relative to controls without lesions. The opposite was true for food-associated environments, for which the rats with STN lesions showed an enhanced preference.

These findings not only seem to be consistent with previous findings linking drug addiction with STN dysfunction, but also suggest the potential for more focused strategies for treating addiction. "These findings have important functional implications," the authors indicate, "as they suggest that different parallel microcircuits mediate responses for natural reward versus cocaine... this possibility opens new perspectives for the development of specific and efficient treatments for drug abuse."

Michael Eisenstein 